\title{
Détermination de la distribution des contraintes dans un matériau oxydé par une méthode de la courbure
}

\author{
J. G. Zhao et A. M. Huntz \\ Laboratoire de Métallurgie Structurale, ISMA CNRS LIA 1107, Université Paris Sud, 91405 Orsay Cedex, \\ France
}

(Reçu le 6 janvier 1987, révisé le 7 juillet 1987, accepté le 9 juillet 1987)

\begin{abstract}
Résumé. - Pour déterminer les contraintes dues à l'oxydation d'un matériau, une nouvelle méthode de courbure a été mise au point. Elle consiste à mesurer l'évolution de la courbure d'un échantillon mince préalablement oxydé au cours d'abrasions successives de l'une de ses grandes faces. Appliquée au système $\mathrm{NiCr}-\mathrm{Cr}_{2} \mathrm{O}_{3}$, elle a permis de tracer les courbes de distribution des contraintes dans la couche oxydée et dans le substrat.

Abstract. - A new curvature method was developed for determining the stress distribution in an oxidized material. This technique consists in measuring the evolution of the curvature of a previously oxidized thin sample during successive sputterings of one of its large faces. It was applied to the $\mathrm{NiCr}-\mathrm{Cr}_{2} \mathrm{O}_{3}$ system and allowed to plot the stress distribution curves in both the oxide scale and the substrate.
\end{abstract}

\section{Introduction.}

Des travaux antérieurs, effectués sur le système $\mathrm{Ni}_{66} \mathrm{Cr}_{34}-\mathrm{Cr}_{2} \mathrm{O}_{3}$ en vue de déterminer les contraintes résiduelles dues à l'oxydation du substrat métallique, ont montré que l'analyse des contraintes par diffraction des rayons $\mathrm{X}$ aux grands angles est très complexe $[1,2]$. En effet, il est difficile de faire la part entre les variations des distances interréticulaires dues aux contraintes et celles dues, par exemple, à des gradients de concentration chimique. C'est pourquoi une nouvelle méthode dite de la «courbure » a été mise au point.

Par la mesure de l'évolution de la courbure d'un échantillon oxydé en fonction de son épaisseur résiduelle, la méthode de courbure permet de déterminer la distribution des contraintes internes dans l'épaisseur de l'échantillon, c'est-à-dire dans la couche d'oxyde et dans le substrat métallique.
Cette méthode a été appliquée, pour la première fois, au système $\mathrm{Ni}_{66} \mathrm{Cr}_{34}-\mathrm{Cr}_{2} \mathrm{O}_{3}$.

\section{Présentation de la méthode de la courbure.}

Cette méthode a été développée à partir de la méthode de la flèche, mise au point par Flavenot [3] et utilisée pour déterminer la distribution de contraintes internes dans des éprouvettes ayant subi une opération de grenaillage. Le principe de cette méthode est rappelé dans la suite et schématisé dans la figure 1.

1.1 MÉthode DE LA FLÈche (cf. Fig. 1). L'éprouvette précontrainte présente une flèche $f$. Par un polissage quelconque, on enlève progressivement les couches superficielles contenant les contraintes résiduelles. L'éprouvette se déforme pour conserver son équilibre, et la flèche présentée 

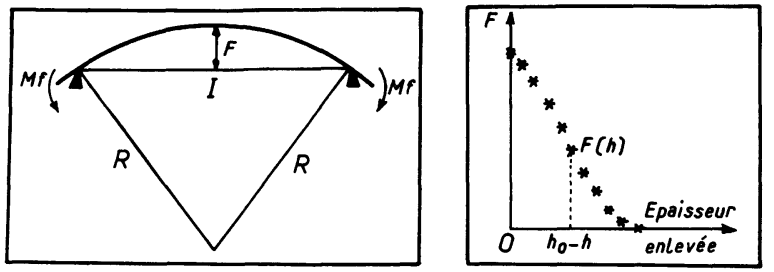

Fig. 1. - Schéma et résultats d'analyse de contraintes sur éprouvettes grenaillées, par la méthode de la « Flèche ", d'après J. F. Flavenot [3].

[Schematization and results of stress analysis on shot peened samples by the arrow method of J. F. Flavenot [3].]

par l'éprouvette varie donc. Cette variation de la flèche est reliée à la distribution des contraintes résiduelles dans l'épaisseur de l'échantillon par la formule :

$$
\begin{aligned}
\sigma(h) & =\frac{4 E}{3 l^{2}} \times \\
\times & \left.\times-h^{2} \frac{\mathrm{d} F}{\mathrm{~d} h}+6 h\left[F\left(h_{0}\right)-F(h)\right]+2 \int_{h_{0}}^{h} e \mathrm{~d} F\right\}
\end{aligned}
$$

où $l$ est la distance entre les deux points d'appui pour mesurer la flèche ; les autres notations sont rassemblées dans le tableau I.

Mais il existe deux difficultés principales pour que cette méthode soit utilisée directement dans le cas des contraintes résiduelles d'oxydation :

1) L'éprouvette précontrainte par oxydation ne présente pas de flèche en raison de la symétrie du système (la couche d'oxyde s'est formée sur toutes les surfaces de l'échantillon);

2) L'épaisseur de la couche d'oxyde est très faible, de l'ordre de quelques $\mu \mathrm{m}$ en général; l'enlèvement progressif de couches d'oxyde est difficile en raison de la précision des mesures expérimentales.

Malgré les difficultés d'utilisation de la méthode de la flèche pour caractériser les contraintes internes d'oxydation, le principe de cette méthode est applible après quelques modifications des formules et conduit à la méthode de la courbure.

1.2 PRINCIPE DE LA MÉTHOde DE LA COURBURE. - Par rapport à la méthode de la «flèche", la méthode de la « courbure » présente trois différences essentielles :

1) L'épaisseur de l'éprouvette est progressivement réduite par abrasion mécanique en conservant toujours une couche d'oxyde sur l'une des grandes faces de l'échantillon. Celle-ci joue le rôle de source de contraintes.
Tableau I. - Notation utilisée dans la méthode de la Courbure.

$\sigma(h)):$ Contrainte interne dans la fibre de cote $h$, $\sigma(x)\}:\left(0 \leqslant h \leqslant h_{0}\right), x$ ou $e$, après oxydation,

$\sigma(e)$ : calculée par le modèle de poutre simple.

$\sigma_{\mathrm{h}}(h)$ : Contrainte résiduelle existant à la surface de l'éprouvette usinée.

$\sigma_{\mathrm{e}}(e)$ : Contrainte résiduelle existant à la surface de l'éprouvette usinée quand $h=e$.

$\sigma_{\mathrm{f}}$ : Contrainte normale due à un effort de flexion.

$\sigma_{\mathrm{n}} \quad$ : Contrainte normale due à une force normale.

$\Delta \sigma \quad$ : Modification du niveau de contrainte interne sur la fibre superficielle considérée due à l'abrasion préliminaire.

$\sigma_{\mathrm{p}}(x)$ : Contrainte résiduelle due à l'effet de polissage mécanique.

$\delta \quad:$ Profondeur des couches influencées par les contraintes internes de polissage.

$\bar{\sigma}_{\mathrm{p}} . \delta$ : Moment de flexion dû aux contraintes de polissage.

$h_{0} \quad:$ Epaisseur initiale de l'éprouvette.

$h \quad$ : Epaisseur résiduelle de l'éprouvette.

$x \quad$ : Repère de Descartes ; sert aussi comme cote des fibres de l'échantillon quand il est plan.

: Variable de l'épaisseur de l'éprouvette.

$e_{0} \quad$ : Fibre neutre.

$b \quad$ : Largeur de l'éprouvette.

$\xi_{\text {ox }} \quad$ : Epaisseur de la couche d'oxyde.

$p \quad$ : Profondeur des couches influencées par les contraintes internes d'oxydation.

$I_{\mathrm{m}} \quad$ : Moment d'inertie de la section droite du substrat de l'éprouvette.

$I_{\mathrm{ox}} \quad$ : Moment d'inertie de la section droite de la couche d'oxyde.

$p \quad: \quad$ Force normale au système

$M \quad$ : Moment de flexion du système.

$F \quad$ : Courbure de l'éprouvette.

$F_{0} \quad$ : Courbure de l'éprouvette avant usinage.

$F_{\mathrm{p}}$ : Courbure de l'échantillon étalon non oxydé, due aux contraintes de polissage.

$E_{\mathrm{m}} \quad$ : Module de Young du matériau.

$E_{\mathrm{ox}} \quad$ : Module de Young de l'oxyde.

2) Le rayon de courbure de l'échantillon est mesuré par un micro-contrôleur électronique $\left({ }^{1}\right)$.

3) Le système est, en réalité, un matériau composite (oxyde-substrat); l'échantillon étant mince $\left(h_{0} \leqslant 0,5 \mathrm{~mm}\right)$, des approximations faites dans la formule de Flavenot doivent être éliminées.

$\left.{ }^{1}\right)$ Dispositif Microcontrôle comprenant deux tables à microdéplacements perpendiculaires, reliées à un translateur affichant, et un microscope équipé d'une caméra vidéo et d'un éclairage annulaire par fibres optiques, relié à un moniteur + écran. 
1.2.1 Considérations liées à la forme droite de l'éprouvette après oxydation et à la distribution symétrique des contraintes internes. - Nous considérons que la couche d'oxyde développée sur les surfaces de l'échantillon joue un rôle de source de contraintes et la distribution des contraintes internes peut être écrite sous la forme générale (Fig. 2) :

$$
\sigma(x)=\left\{\begin{array}{cl}
\sigma(x) & 0 \leqslant x<p \\
0 & p \leqslant x \leqslant h_{0}-p \\
\sigma(x) & h_{0}-p<x \leqslant h_{0}
\end{array}\right\}
$$

et

$$
\left.\begin{array}{l}
M=\int_{0}^{h_{0}} \sigma(x)\left(x-x_{0}\right) \cdot b \cdot \mathrm{d} x \\
P=\int_{0}^{h_{0}} \sigma(x) \cdot b \cdot \mathrm{d} x
\end{array}\right\}
$$

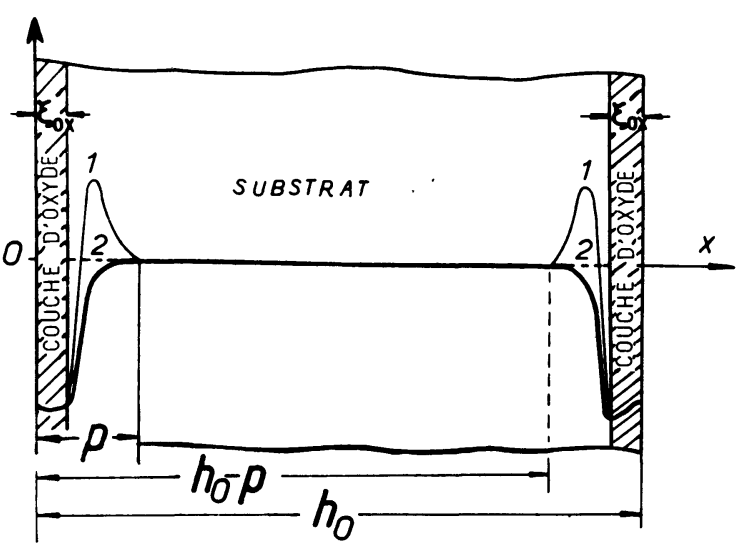

Fig. 2. - Schématisation de la distribution symétrique des contraintes internes d'oxydation et signification des symboles $\xi_{\text {ox }}, p, h_{0}$.

[Scheme of the symmetrical distribution of the oxidation internal stresses and illustration of the $\xi_{\mathrm{ox}}, p, h_{0}$ symbols.]

En considérant que le système oxyde-substrat se comporte comme une poutre simple, c'est-à-dire que la poutre se déforme sous un moment de flexion $M$ constant, nous avons [4] :

$M=\frac{1}{R}\left(E_{\mathrm{m}} I_{\mathrm{m}}+E_{\mathrm{ox}} I_{\mathrm{ox}}\right)=F\left(E_{\mathrm{m}} I_{\mathrm{m}}+E_{\mathrm{ox}} I_{\mathrm{ox}}\right)$.

D'après (1) à (3) :

$$
\begin{gathered}
\int_{0}^{p} \sigma(x)\left(x-x_{0}\right) \cdot b \cdot \mathrm{d} x+\int_{h_{0}-p}^{h_{0}} \sigma(x)\left(x-x_{0}\right) \times \\
\times b \cdot \mathrm{d} x=F\left(E_{\mathrm{m}} I_{\mathrm{m}}+E_{\mathrm{ox}} I_{\mathrm{ox}}\right) .
\end{gathered}
$$

En supposant que la distribution des contraintes internes est symétrique, nous vérifions que le moment de flexion $M$ est nul et que $F=0$, quel que soit le niveau des contraintes internes. L'éprouvette se tient sous forme plane : c'est le cas de l'éprouvette juste après oxydation.
1.2.2 Apparition de la courbure. - En revanche, si on enlève une couche d'oxyde de l'une des deux surfaces, la symétrie ne se maintient plus, l'éprouvette va se déformer. Plus l'épaisseur résiduelle est mince, plus la courbure de l'échantillon est marquée.

$\mathrm{Si}$ on suit la variation de la courbure $F$ en diminuant progressivement l'épaisseur de l'échantillon, on peut atteindre la distribution des contraintes internes par deux méthodes que nous allons maintenant présenter.

\section{Relations entre la courbure $F$ et la distribution des contraintes.}

2.1 LE MATÉRIAU EST CONSIDÉRÉ COMME HOMOGÈNE. - Ce qui impose $E_{\mathrm{ox}} \sim E_{\mathrm{m}}, e_{0}=h / 2$ et $\xi_{\text {ox }} \leqslant h$, le calcul est effectué dans l'hypothèse d'une poutre en flexion, en respectant les définitions conventionnelles des signes des sollicitations mécaniques [4] :

$$
\left\{\begin{array}{l}
M=\int_{0}^{h} \sigma(e)\left(e-\frac{h}{2}\right) \cdot b \cdot \mathrm{d} e \\
M=E_{\mathrm{m}} \frac{b h^{3}}{12 R}=E_{\mathrm{m}} \cdot \frac{b h^{3}}{12} \cdot F
\end{array}\right\}
$$$$
\text { puisque } \quad I_{\mathrm{m}}=\frac{b h^{3}}{12} \quad[3] \text {. }
$$

Supposons maintenant que, lorsque l'épaisseur résiduelle de l'échantillon est $h$, on enlève une couche mince d'épaisseur $\mathrm{d} h(\mathrm{~d} h>0)$ dans le côté concave ; cette couche subissait, avant d'être enlevée, une contrainte superficielle $\sigma_{\mathrm{h}}(h)$, donc une force normale $\mathrm{d} P=\sigma_{\mathrm{h}}(h) \cdot b \cdot \mathrm{d} h$, et un moment $\mathrm{d} M=\sigma_{\mathrm{h}}(h) \cdot b \cdot \mathrm{d} h \cdot \frac{h}{2}$.

$$
\begin{aligned}
& \text { Or } \mathrm{d} M=E_{\mathrm{m}} \frac{b}{12}\left(h^{3} \mathrm{~d} F+3 F h^{2} \mathrm{~d} h\right), \\
& \operatorname{donc}_{\mathrm{h}}(h) \cdot b \cdot \mathrm{d} h \cdot \frac{h}{2}=\frac{E_{\mathrm{m}}}{12} b\left(h^{3} \mathrm{~d} F+3 F h^{2} \mathrm{~d} h\right) \\
& \text { soit, } \quad \sigma_{\mathrm{h}}(h)=\frac{E_{\mathrm{m}}}{6}\left(h^{2} \frac{\mathrm{d} F}{\mathrm{~d} h}+3 h F\right) .
\end{aligned}
$$

Mais cette valeur n'est pas la contrainte résiduelle après oxydation. En effet, l'enlèvement des couches précédentes a déjà modifié la distribution des contraintes. Pour déterminer les contraintes internes qui existaient dans l'éprouvette avant abrasion, la somme des modifications des contraintes internes $\Delta \sigma$, dans. cette fibre considérée, doit être prise en compte. Nous allons calculer $\Delta \sigma$.

Plaçons-nous dans le cas où l'épaisseur de l'échantillon est $e\left(h<e<h_{0}\right)$; une couche de matériau d'épaisseur $\mathrm{d} e(\mathrm{~d} e>0)$, qui subissait une contrainte superficielle $\sigma_{\mathrm{e}}(e)$, a déjà été abrasée. Cette abra- 
sion a induit un moment de flexion : $\mathrm{d} M=$ $\sigma_{\mathrm{e}}(e) \cdot b \cdot \frac{e}{2} \cdot \mathrm{d} e$ et une force normale $\mathrm{d} P=$ $\sigma_{\mathrm{e}}(e) \cdot b . \mathrm{d} e$. La modification des contraintes résiduelles dans la fibre de côte $(e-h)$ est (Fig. 3) :

$$
\mathrm{d} \sigma=\mathrm{d} \sigma_{\mathrm{n}}+\mathrm{d} \sigma_{\mathrm{f}}
$$

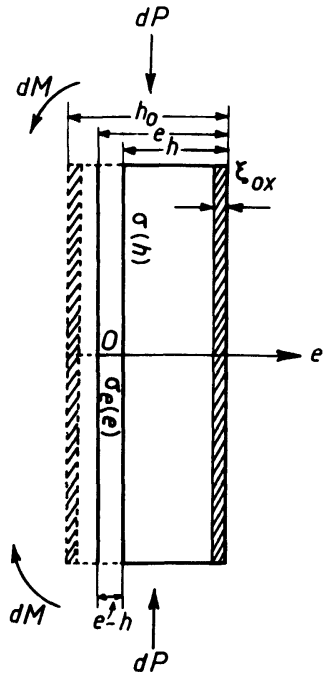

(A)

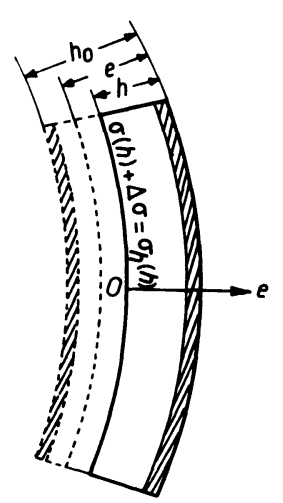

(B)
Fig. 3. - Schéma représentant la variation de la distribution des contraintes internes due à l'enlèvement de matière par abrasions successives.

[Scheme showing the variation of the internal stress distribution due to the successive sputterings.]

$\mathrm{d} \sigma_{\mathrm{n}}$ étant une modification homogène des contraintes dans l'épaisseur de l'échantillon :

$$
\mathrm{d} \sigma_{\mathrm{n}}=\frac{\mathrm{d} P}{b \cdot e}=\sigma_{\mathrm{e}}(e) \frac{\mathrm{d} e}{e}
$$

et $\mathrm{d} \sigma_{\mathrm{f}}$, une modification de distribution linéaire des contraintes due au changement de courbure :

$$
\mathrm{d} \sigma_{\mathrm{f}}=E_{\mathrm{m}}\left[(e-h)-\frac{e}{2}\right] \mathrm{d} F
$$

avec la formule (6) on peut écrire :

$$
\mathrm{d} F=\left(\frac{6}{E_{\mathrm{m}}} \cdot \sigma_{\mathrm{e}}(e)-3 e F\right) \frac{\mathrm{d} e}{e^{2}}
$$

soit

$$
\begin{aligned}
\mathrm{d} \sigma_{\mathrm{f}} & =E_{\mathrm{m}}\left(\frac{e}{2}-h\right)\left(\frac{6}{E_{\mathrm{m}}} \cdot \sigma_{\mathrm{e}}(e)-3 e f\right) \frac{\mathrm{d} e}{e^{2}} \\
& =\sigma_{\mathrm{e}}(e)\left(3-\frac{6 h}{e}\right) \frac{\mathrm{d} e}{e}-3 E_{\mathrm{m}} F\left(\frac{e}{2}-h\right) \frac{\mathrm{d} e}{e}
\end{aligned}
$$

d'où

$$
\begin{aligned}
\mathrm{d} \sigma & =\sigma_{\mathrm{e}}(e) \frac{\mathrm{d} e}{e}+\sigma_{\mathrm{e}}(e)\left(3-\frac{6 h}{e}\right) \frac{\mathrm{d} e}{e} \\
& -3 E_{\mathrm{m}} F\left(\frac{e}{2}-h\right) \frac{\mathrm{d} e}{e} \\
= & \sigma_{\mathrm{e}}(e)\left(4-\frac{6 h}{e}\right) \frac{\mathrm{d} e}{e}-3 E_{\mathrm{m}} F\left(\frac{e}{2}-h\right) \frac{\mathrm{d} e}{e} .
\end{aligned}
$$

Donc, la somme des modifications des contraintes internes $\Delta \sigma=\int_{h}^{h_{0}} \mathrm{~d} \sigma$, dans la fibre $h$, est d'après (6) et (7) :

$\Delta \sigma=\frac{2}{3} E_{\mathrm{m}} \int_{h}^{h_{0}} e \mathrm{~d} F+\frac{E_{\mathrm{m}}}{2} \int_{h}^{h_{0}} F \mathrm{~d} e-E_{\mathrm{m}} h \int_{h}^{h_{0}} \mathrm{~d} F$.

Pour déterminer les contraintes résiduelles avant l'opération d'abrasion, il faut soustraire de la contrainte superficielle $\sigma_{\mathrm{h}}(h)$ cette modification $\Delta \sigma$ (cf. Fig. 3).

$$
\sigma(h)=\sigma_{\mathrm{h}}(h)-\Delta \sigma
$$

et comme $F\left(h_{0}\right)=0$ et

$$
-\int_{h}^{h_{0}} e \mathrm{~d} F=h F+\int_{h}^{h_{0}} F \cdot \mathrm{d} e,
$$

nous avons finalement :

$$
\sigma(h)=\frac{E_{\mathrm{m}}}{6} \cdot\left(h^{2} \cdot \frac{\mathrm{d} F}{\mathrm{~d} h}+\int_{h}^{h_{0}} F \cdot \mathrm{d} e+h \cdot F\right)
$$

c'est-à-dire que la contrainte interne dans la fibre $\left(h_{0}-h\right)$, après oxydation, est déterminée par la pente de la courbe $F=f(e)$ et la surface hachurée dans la figure 4.

2.2 MATÉRIAU CONSIDÉRÉ COMME COMPOSITE $\left(E_{\mathrm{ox}} \neq E_{\mathrm{m}}\right)$. - A partir des équations de base (1-3), nous avons :

$$
\int_{0}^{h} \sigma(e)\left(e-e_{0}\right) \cdot b \cdot \mathrm{d} e=F\left(E_{\mathrm{m}} \cdot I_{\mathrm{m}}+E_{\mathrm{ox}} \cdot I_{\mathrm{ox}}\right) \text {. }
$$

Si l'on note $G(h)=\int_{0}^{h} \sigma(e) \cdot\left(e-e_{0}\right) \cdot b$. de et $Q(h)=F\left(E_{\mathrm{m}} \cdot I_{\mathrm{m}}+E_{\mathrm{ox}} \cdot I_{\mathrm{ox}}\right), G(h)$ et $Q(h)$ étant deux fonctions continues de $h$, égales pour toute valeur de la variable $h$, il s'ensuit :

$$
G^{\prime}(h)=Q^{\prime}(h) \text {. }
$$




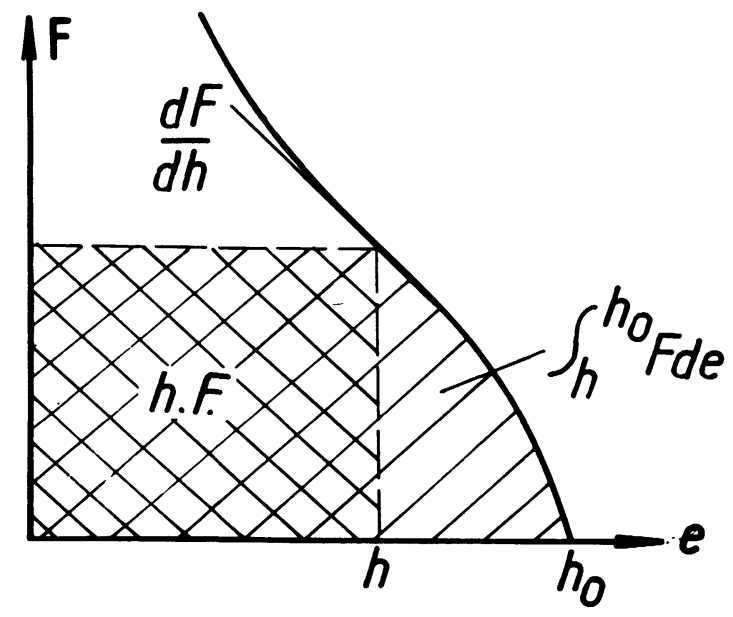

Fig. 4. - Schéma montrant les grandeurs à calculer sur la courbe de variation de la courbure pour déterminer la contrainte en un point $h$ avec la formule (9) (matériau homogène $\equiv$ poutre en flexion).

[Scheme showing on a curvature variation curve the parameters which have to be calculated for determining the stress at a depth $h$ using the formula (9) (homogeneous material).]

\section{Comme}

$$
G^{\prime}(h)=b\left[\sigma(h)\left(h-e_{0}\right)-\frac{\mathrm{d} e_{0}}{\mathrm{~d} h} \int_{0}^{h} \sigma(e) \cdot \mathrm{d} e\right]
$$

et

$$
\begin{aligned}
Q^{\prime}(h)=F\left(E_{\mathrm{m}} \cdot \frac{\mathrm{d} I_{\mathrm{m}}}{\mathrm{d} h}+E_{\mathrm{ox}}\right. & \left.\cdot \frac{\mathrm{d} I_{\mathrm{ox}}}{\mathrm{d} h}\right)+ \\
& +\frac{\mathrm{d} F}{\mathrm{~d} h}\left(E_{\mathrm{m}} \cdot I_{\mathrm{m}}+E_{\mathrm{ox}} \cdot I_{\mathrm{ox}}\right)
\end{aligned}
$$

nous obtenons

$$
\begin{aligned}
\sigma(h)=\frac{1}{h-e_{0}} & \frac{\mathrm{d} e_{0}}{\mathrm{~d} h} \int_{0}^{h} \sigma(e) \cdot \mathrm{d} e+ \\
& +g\left(E_{\mathrm{m}}, E_{\mathrm{ox}}, h, \xi_{\mathrm{ox}}, F, \frac{\mathrm{d} F}{\mathrm{~d} h}\right)
\end{aligned}
$$

avec

$$
\begin{aligned}
g\left(E_{\mathrm{m}}, E_{\mathrm{ox}}, I_{\mathrm{m}}, I_{\mathrm{ox}}, F, \frac{\mathrm{d} F}{\mathrm{~d} h}, \xi_{\mathrm{ox}}\right)= \\
=\frac{Q^{\prime}(h)}{b\left(h-e_{0}\right)}=F \cdot A(h)+\frac{\mathrm{d} F}{\mathrm{~d} h} \cdot B(h) \\
A(h)=\frac{E_{\mathrm{m}}}{h-e_{0}}\left(\frac{1}{b} \cdot \frac{\mathrm{d} I_{\mathrm{m}}}{\mathrm{d} h}+r \cdot \frac{1}{b} \cdot \frac{\mathrm{d} I_{\mathrm{ox}}}{\mathrm{d} h}\right) \\
B(h)=\frac{E_{\mathrm{m}}}{h-e_{0}}\left(\frac{1}{b} I_{\mathrm{m}}+r \cdot \frac{1}{b} \cdot I_{\mathrm{ox}}\right) \\
r \quad=E_{\mathrm{ox}} / E_{\mathrm{m}} .
\end{aligned}
$$

Introduisons la variable réduite :

$$
q=\left(h-\xi_{\text {ox }}\right) /\left(r \cdot \xi_{\text {ox }}\right) .
$$

Les moments d'inertie des sections droites du substrat et de l'oxyde valent :

$$
\left\{\begin{aligned}
I_{\mathrm{m}}= & \frac{b}{3}\left[\left(h-\xi_{\mathrm{ox}}\right)^{3}-3\left(h-\xi_{\mathrm{ox}}\right)^{2} e_{0}\right. \\
& \left.+3\left(h-\xi_{\mathrm{ox}}\right) e_{0}^{2}\right] \\
I_{\mathrm{ox}}= & \frac{b}{3}\left[3\left(h-e_{0}\right)^{2} \xi_{\mathrm{ox}}-3\left(h-e_{0}\right) \xi_{\mathrm{ox}}^{2}+\xi_{\mathrm{ox}}^{3}\right]
\end{aligned}\right.
$$

d'où

$$
\left\{\begin{array}{l}
\frac{\mathrm{d} I_{\mathrm{m}}}{\mathrm{d} h}=b\left[\left(1-\frac{\mathrm{d} e_{0}}{\mathrm{~d} h}\right)\left(h-\xi_{\mathrm{ox}}-e_{0}\right)^{2}+e_{0}^{2} \frac{\mathrm{d} e_{0}}{\mathrm{~d} h}\right] \\
\frac{\mathrm{d} I_{\mathrm{ox}}}{\mathrm{d} h}=b\left(1-\frac{\mathrm{d} e_{0}}{\mathrm{~d} h}\right)\left[2 \xi_{\mathrm{ox}}\left(h-e_{0}\right)-\xi_{\mathrm{ox}}^{2}\right]
\end{array}\right.
$$

avec

$$
\begin{aligned}
e_{0} & =\frac{1}{2}\left(h-\xi_{\mathrm{ox}}+\frac{h}{1+q}\right) \\
\frac{\mathrm{d} e_{0}}{\mathrm{~d} h} & =\frac{1}{2}\left[1+\frac{1}{1+q}-\frac{h / r \cdot \xi_{\mathrm{ox}}}{(1+q)^{2}}\right] .
\end{aligned}
$$

C'est-à-dire que la contrainte résiduelle, dans ùne fibre de côte $h$, est déterminée par la vitesse de variation de $F$, la valeur de $F$ en ce point et la distribution générale des contraintes internes.

Dans ces deux façons de considérer le système, nous avons supposé que la courbure de l'échantillon est constante (forme circulaire) et que la contrainte interne n'est qu'une fonction de la cote $e$ des fibres : les forces normales n'interviennent pas sur la déformation de l'échantillon. En réalité, notre système n'est pas exactement dans ce cas :

$$
S=\frac{1}{h_{0}} \int_{h}^{h_{0}} \sigma(h) \mathrm{d} h
$$

(dans le cas d'une poutre simple, $S=0$ ) et nous effectuons la correction :

$$
\sigma(x)=\sigma(h)-S \quad(x=h) .
$$

Après cette correction, on vérifie facilement que les contraintes ainsi calculées obéissent bien aux lois de l'équilibre mécanique relatives aux formes normales et au moment de flexion.

2.3 COMPARAISON DES DEUX FORMULATIONS (Rel. (9) et (11)). - Les deux formulations sont issues d'une même base, la mécanique de flexion d'une poutre simple; mais il y a des différences sensibles entre celles-ci :

(1) La formule (9) tient compte de la déformation à chaque instant ; la mécanique est utilisée tout au long de la démonstration ; tandis que la formule (11) ne fait appel qu'à une propriété mathématique des fonctions $G$ et $Q$. 
(2) La formule (9) implique une variation monotone de la courbure $F$; cette variation correspond à celle imposée par un polissage électrolytique; au contraire, la formule (11) implique que la distribution $\sigma(e)$ est invariante quelle que soit l'épaisseur résiduelle. Basée sur la loi de superposition du champ de contraintes, cette formule demande que l'échantillon se déforme de façon parfaitement élastique. Elle est donc bien adaptée au polissage mécanique puisque, dans ce cas, à chaque nouvelle abrasion, on impose à l'échantillon d'être plan (Fig. 5).

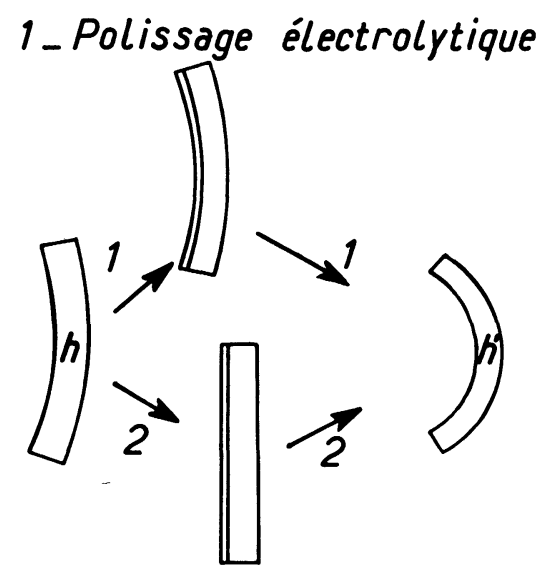

\section{2_Polissage mécanique}

Fig. 5. - Schéma montrant l'effet de polissage divers : si on enlève une couche superficielle de matière par un polissage électrolytique, la courbure de l'échantillon varie de façon continue ; par un polissage mécanique, la courbure de l'échantillon varie de façon discontinue.

[Scheme of the effects of various polishings : by electrochemical polishing of a superficial layer, the sample curvature continuously varies, whereas it discontinuously varies by mechanical polishing.]

\section{Problèmes posés par l'application de cette méthode.}

\subsection{Problèmes RÉSOLUS GRÂCE À L'HYPOTHÈSE} DE LA DISTRIBUTION SYMÉTRIQUE. - Selon l'équation (10), quand $h$ tend vers zéro, $I$ tend vers zéro et la courbure de l'échantillon $F$ ne sera plus mesurable car elle devient trop élevée. Avec le microcontrôleur électronique, nous arrivons seulement à mesurer la courbure d'échantillons d'épaisseur supérieure à $60 \mu \mathrm{m}$ (cette valeur dépend aussi de la nature du matériau). Ceci implique qu'on ne peut déterminer la distribution des contraintes dans toute l'épaisseur de l'échantillon. De plus, lorsque l'éprouvette est très mince, on risque de la déformer; or, ni la formule (9), ni la formule (11) ne peuvent être utilisées s'il y a déformation plastique de l'éprouvette.
Mais, avec une distribution symétrique, nous avons expliqué pourquoi l'échantillon, après oxydation, reste sous forme plane malgré la présence de contraintes internes. Cette hypothèse est encore utile pour déterminer la distribution des contraintes dans toute l'épaisseur de l'échantillon. En effet, avec cette hypothèse, nous avons besoin seulement d'analyser la moitié de la courbe, et l'autre partie sera déterminée par la relation $\sigma(x)=\sigma\left(h_{0}-x\right)$.

En réalité, l'échantillon n'est pas exactement plan après oxydation. Le microcontrôleur analyse la perturbation de profil du contour de l'échantillon comme une courbure. C'est pourquoi nous avons trouvé expérimentalement que $F\left(h_{0}\right) \neq 0$ (Fig. 6). En tenant compte de l'hypothèse de la distribution symétrique, nous avons donc utilisé, comme valeur de la courbure, une valeur corrigée : $F(h)=$ $F(h)-F\left(h_{0}\right)$, et la formule (11) s'écrit alors :

$$
\begin{aligned}
\sigma(h)= & \frac{1}{h-e_{0}} \frac{\mathrm{d} e_{0}}{\mathrm{~d} h} \times \\
\times & {\left[\int_{0}^{h_{0}-h} \sigma\left(h_{0}-e\right) \mathrm{d} e+2 \int_{h_{0} / 2}^{h} \sigma(e) \mathrm{d} e\right]+} \\
& +g\left(E_{\mathrm{m}}, E_{\mathrm{ox}}, h, \xi_{\mathrm{ox}}, F, \frac{\mathrm{d} F}{\mathrm{~d} h}\right) \cdot
\end{aligned}
$$

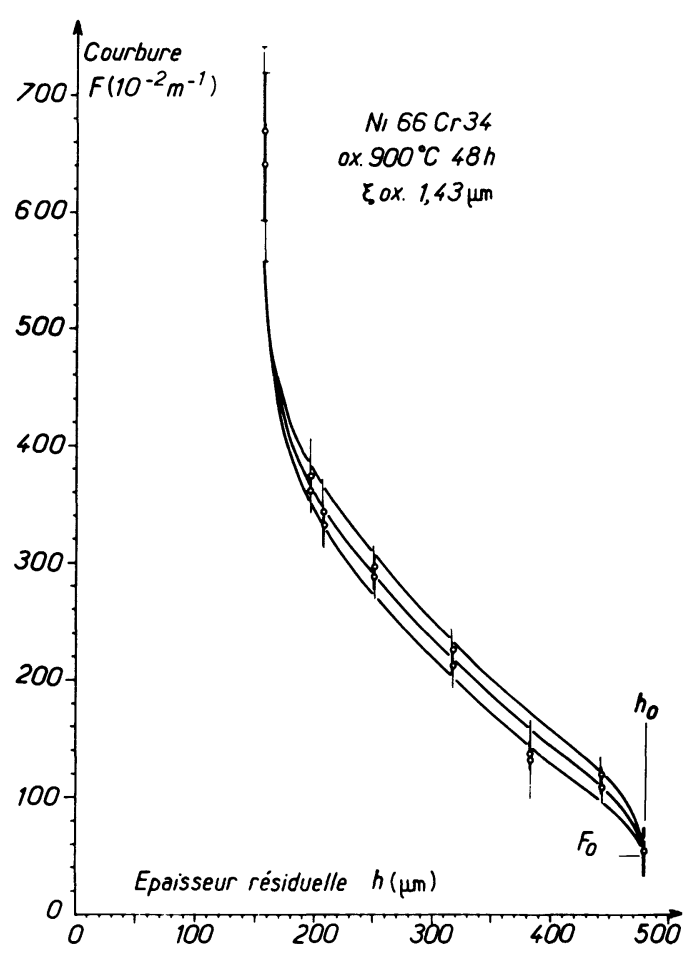

Fig. 6. - Courbe expérimentale de la variation de la courbure en fonction de l'épaisseur résiduelle de l'échantillon.

[Experimental curve of the curvature variation vs. the sample residual thickness.] 
Cette équation a été résolue par la méthode dite "self-consistent», qui nous donne une solution numérique. La première valeur de $\sigma$ a été prise égale à 0 et la précision du calcul est d'un MPa. L'organigramme de calcul pour les formules (9) et (13) est présenté dans le tableau II et les résultats d'expérience dans la figure 7.

Tableau II. - Tableau représentant le processus de détermination des contraintes internes par la méthode de courbure.

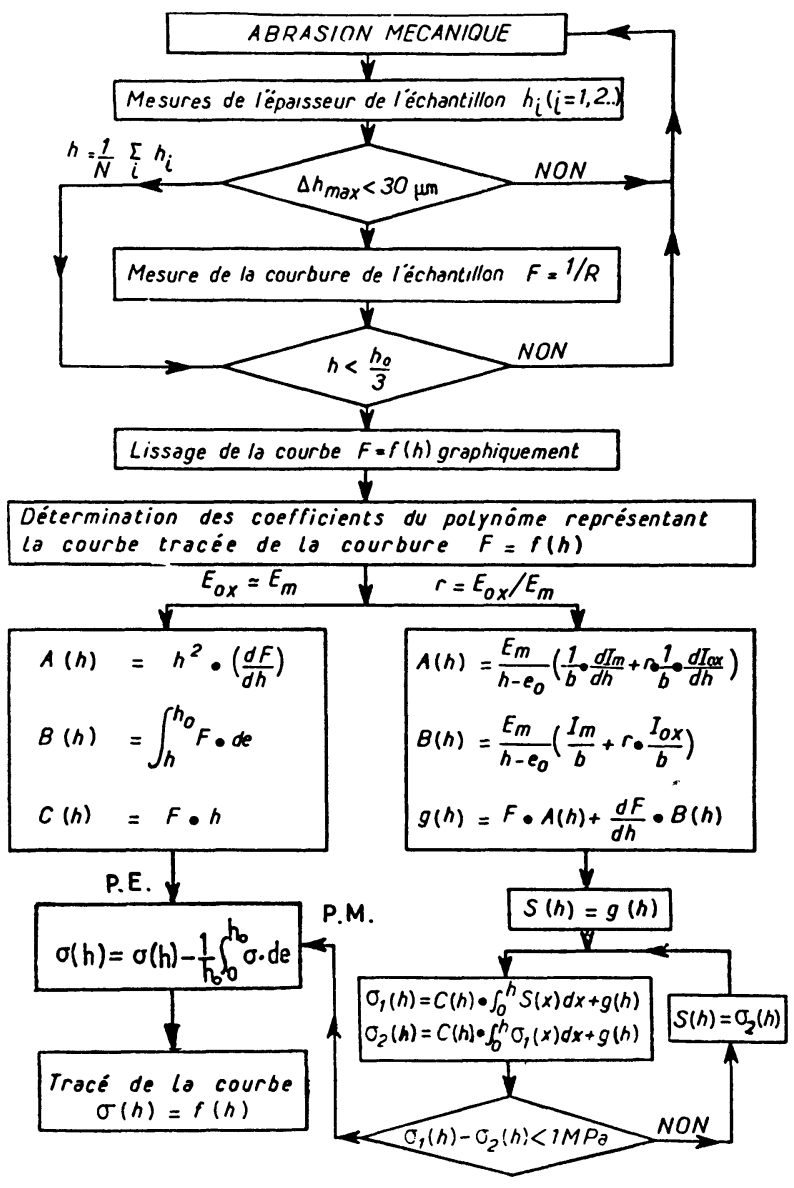

\subsection{DÉTERMINATION DE LA CONTRAINTE INTERNE} DANS LA COUCHE D'OXYDE. - Les résultats de la méthode de la courbure (Fig. 7) montrent une distribution continue des contraintes internes dans toute l'épaisseur de l'éprouvette. En plus, cette distribution continue donne l'impression que les contraintes à l'interface externe $\sigma(0)$ sont supérieures aux contraintes $\sigma\left(\xi_{\text {ox }}\right)$ à l'interface interne M-O. Ceci est difficile à concevoir. Nous considérons que ce résultat provient, d'une part du lissage des courbes expérimentales de courbure par une fonction continue, d'autre part, du fait que l'épaisseur de la première couche enlevée est supérieure à l'épaisseur de la couche d'oxyde (cf. Fig. 6). (Rappelons que $\xi_{\text {ox }}$ est faible dans le cas des alliages $\mathrm{Ni}_{66} \mathrm{Cr}_{34}$.) Compte tenu de cette difficulté actuelle, nous proposons un paramètre $\bar{\sigma}_{\text {ox }}$ qui est la moyenne du niveau des contraintes dans la couche d'oxyde, déterminée par la relation :

$$
\bar{\sigma}_{\text {ox }}=\frac{1}{\xi_{\text {ox }}} \int_{0}^{\xi_{\text {ox }}} \sigma(x) \mathrm{d} x
$$

comme le montre la figure 8 .

3.3 INFLUENCE DES CONTRAINTES DE POLISSAGE. - Dans les paragraphes et les relations précédentes, nous n'avons pas considéré l'influence des contraintes de polissage. Or, il est évident que, dans le cas du polissage mécanique du moins, les contraintes de polissage peuvent modifier les résultats.

Le niveau de contraintes crées par le polissage dépend de beaucoup de paramètres : le grade de papier SiC utilisé, la vitesse d'abrasion, le sens de polissage, la profondeur abrasée à chaque fois [5]. La figure 9 montre, par exemple, l'influence du sens de polissage sur la variation de la courbure de l'échantillon. Nous constatons que les contraintes de polissage se manifestent de façon plus importante quand le sens de polissage est transversal que lorsqu'il est longitudinal. Si le polissage transversal est suivi par un polissage longitudinal, la valeur de $F$ est peu diminuée ; dans le cas inverse (longitudinal + transversal), nous observons une forte augmentation de $F$. Ainsi, la méthode de la courbure exige des échantillons polis dans le même sens, longitudinal, toutes conditions identiques par ailleurs. En comparant la figure 6 et la figure 10 , nous constatons que le rôle des contraintes de polissage (polissage longitudinal) n'est pas très important. Cependant, ce n'est pas le cas pour les échantillons avec de faibles contraintes internes d'oxydation ; une correction est alors indispensable.

La méthode que nous avons utilisée consiste à prendre un échantillon étalon : recuit sous vide, sans oxydation $\left(\xi_{\mathrm{ox}}=0\right)$, toutes conditions identiques par ailleurs à celles utilisées pour les échantillons oxydés. Nous considérons que la profondeur influencée par les contraintes de polissage $(\delta)(<10 \mu \mathrm{m})$ est inférieure à l'épaisseur enlevée chaque fois $(\Delta h)$ $(\approx 10 \mu \mathrm{m})$. Dans ce cas, l'état de contraintes de polissage est toujours le même au cours des abrasions successives et la courbure de l'échantillon étalon augmente seulement du fait de la diminution de l'épaisseur de l'échantillon (Fig. 10). Nous constatons que l'échantillon étalon se déforme sous l'effet de la source de contraintes de polissage qui crée des compressions, selon la relation :

$$
\int_{0}^{\delta} \sigma_{\mathrm{p}}(x)\left(x-\frac{h}{2}\right) \cdot b \cdot \mathrm{d} x=E_{\mathrm{m}} \frac{b h^{3}}{12} F_{\mathrm{p}}
$$

comme $\delta \leqslant h / 2$, nous avons :

$$
M \simeq-\frac{h}{2} \int_{0}^{\delta} \sigma_{\mathrm{p}} b \mathrm{~d} x=-\frac{h}{2} b \bar{\sigma}_{\mathrm{p}} \delta
$$



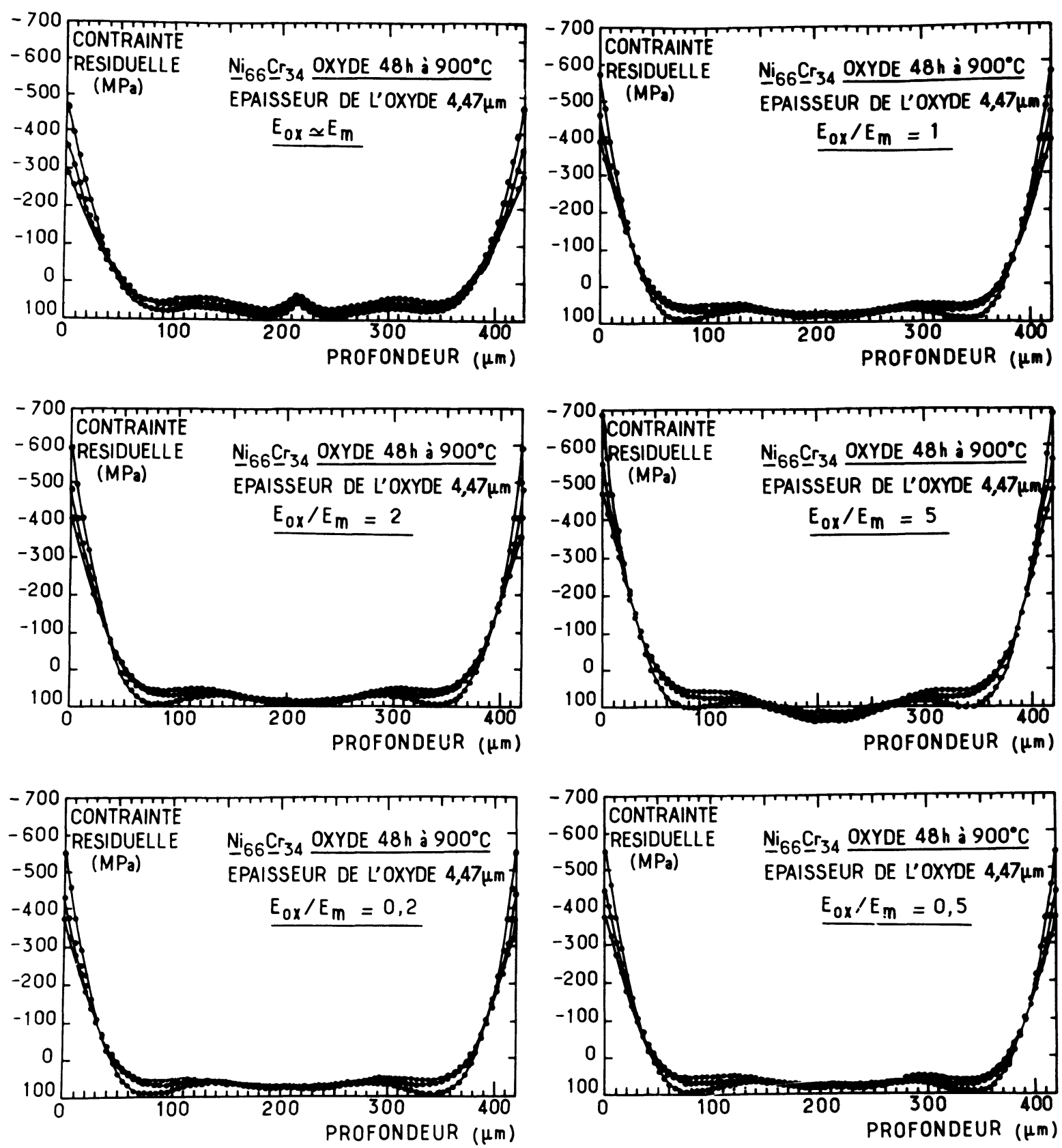

Fig. 7. - Distribution des contraintes dans l'épaisseur d'un échantillon $\mathrm{Ni}_{66} \mathrm{Cr}_{34}$ oxydé $48 \mathrm{~h}$ à $900^{\circ} \mathrm{C}$. La première figure, en haut à gauche, correspond au résultat obtenu avec la formule (9) (matériau homogène $\equiv$ poutre en flexion). Les autres figures correspondent aux calculs issus de la formule (13) (matériau hétérogène), avec diverses valeurs du rapport des modules d'Young, $r=E_{\mathrm{ox}} / E_{\mathrm{m}}$, choisies arbitrairement égales à $1,2,5,0,2$, ou 0,5 et portées en haut à droite des figures. Les différentes courbes dans chaque figure correspondent aux diverses courbes de lissage tracées sur la courbe $F=f(h)$ (cf. Fig. 6).

[Stress distribution curves in a $\mathrm{Ni}_{66} \mathrm{Cr}_{34}$ sample oxidized for $48 \mathrm{~h}$ at $900^{\circ} \mathrm{C}$. The first figure, where $E_{\mathrm{ox}} \sim E_{\mathrm{m}}$, was obtained with the formule (9) (homogeneous material), whereas all the others correspond to the results obtained with equation (13) (heterogeneous material), with various values of the Young modulus ratio $E_{\mathrm{ox}} / E_{\mathrm{m}}$ (value noted on each figure). For each figure the different curves are due to the various smoothing curves plotted for the $F$ variation (cf. Fig. 6).]

d'où :

$$
\bar{\sigma}_{\mathrm{p}} \delta=\int_{0}^{\delta} \sigma_{\mathrm{p}}(x) \mathrm{d} x=-\frac{E_{\mathrm{m}}}{6} F_{\mathrm{p}} h^{2} .
$$

Par la méthode des moindres carrés, la valeur $\bar{\sigma}_{\mathrm{p}} \cdot \delta$ est déterminée sur l'échantillon et une valeur supplémentaire égale à $F_{\mathrm{p}}$ doit être ajoutée à chaque mesure expérimentale de $F$ effectuée sur les échantillons oxydés puis abrasés sur une face (voir un exemple de résultats en annexe I). Les résultats 


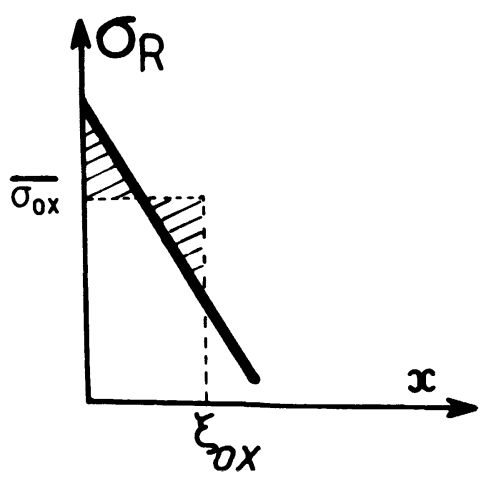

Fig. 8. - Schéma représentant la méthode de détermination de la contrainte interne moyenne dans la couche d'oxyde.

[Scheme showing how the average stress in the oxide scale is determined.]

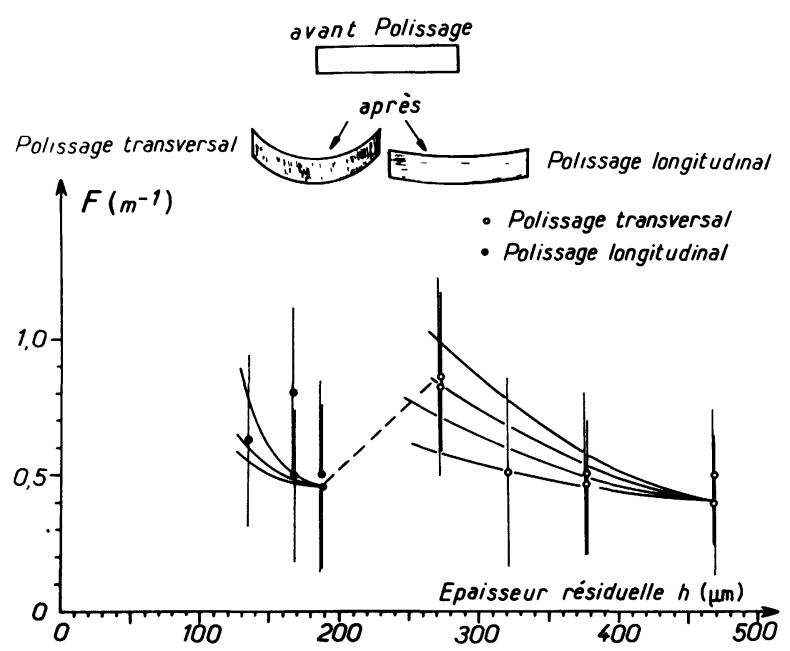

Fig. 9. - Schématisation du rôle du polissage en fonction du sens dans lequel il est effectué.

[Schematization of the polishing effect according to the sample polishing direction.]

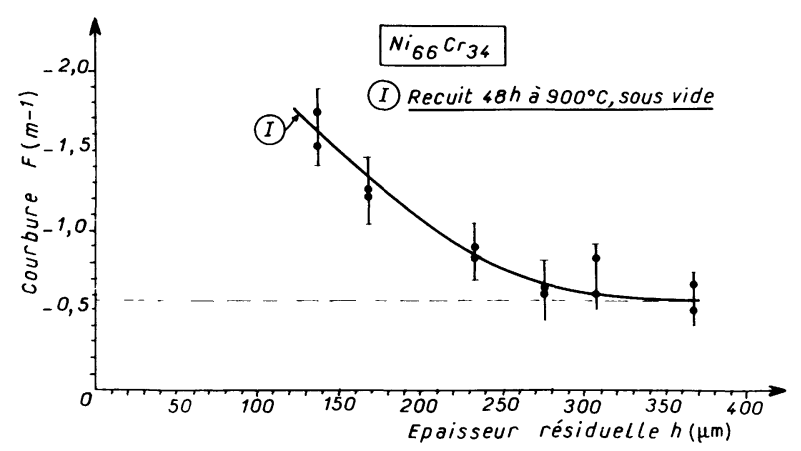

Fig. 10. - Variation de la courbure en fonction de l'épaisseur résiduelle pour un échantillon étalon, non oxydé (recuit $48 \mathrm{~h}$ à $900^{\circ} \mathrm{C}$ sous vide).

[Curvature variation $v s$. the residual thickness of a standard unoxidized sample.]

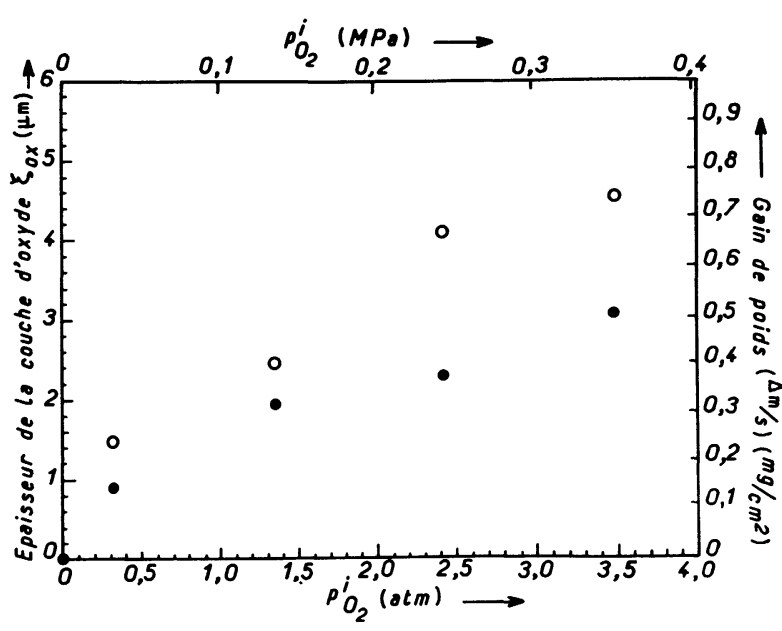

Fig. 11. - Variation de l'épaisseur de la couche d'oxyde $\mathrm{Cr}_{2} \mathrm{O}_{3}$ développée sur l'alliage $\mathrm{Ni}_{66} \mathrm{Cr}_{34}$ oxydé $26 \mathrm{~h}$ ou $48 \mathrm{~h}$, à $900^{\circ} \mathrm{C}$, en fonction de la pression d'oxygène.

[Variation of the thickness of the $\mathrm{Cr}_{2} \mathrm{O}_{3}$ scale developed on $\mathrm{Ni}_{66} \mathrm{Cr}_{34}$ samples oxidized for 26 or $48 \mathrm{~h}$ at $900^{\circ} \mathrm{C}$ with various oxygen pressure.]

présentés dans les figures 7,12 et 13 tiennent compte de cette correction.

3.4 INCERTITUDE DES MESURES. - Les incertitudes les plus importantes sont dues à :

— L'hétérogénéité de l'épaisseur de l'échantillon: dans les meilleures conditions de polissage, les différences moyennes de mesures de $h$ sont de l'ordre de 10 à $20 \mu \mathrm{m}$, ce qui conduit à une incertitude de $30 \%$ sur $F$.

- La courbure de l'échantillon ne suit pas rigoureusement un cercle; les différences moyennes de mesure sont de l'ordre de $10^{-1} \mathrm{~m}^{-1}$, soit $10 \%$ environ. Cette incertitude vient probablement aussi de l'hétérogénéité d'épaisseur de l'échantillon.

- Comme la barre d'erreur sur $F$ est assez importante, il est difficile de décider de l'allure exacte de la courbe de lissage. Les résultats expérimentaux montrent que différentes courbes de lissage conduisent à des valeurs de contraintes différentes, surtout à la surface des échantillons (cf. Figs. 6 et 7).

Finalement, l'incertitude de mesure totale est estimée du même ordre de grandeur que la dispersion des résultats dans les courbes de variation de $\sigma$ en fonction de $h$ (cf. Figs. 7); elle est de $20 \%$ environ en valeur relative.

\section{Résultats expérimentaux.}

Nous avons étudié l'évolution de la distribution des contraintes internes d'oxydation par la méthode de courbure pour des échantillons $\mathrm{Ni}_{66} \mathrm{Cr}_{34}(40 \times 9 \times$ $0,15 \mathrm{~mm}$ ) oxydés à $900^{\circ} \mathrm{C}$, soit pendant $26 \mathrm{~h}$, soit pendant $48 \mathrm{~h}$ [6]. Les résultats d'oxydation sont présentés sur la figure 11 et certaines des courbes de 

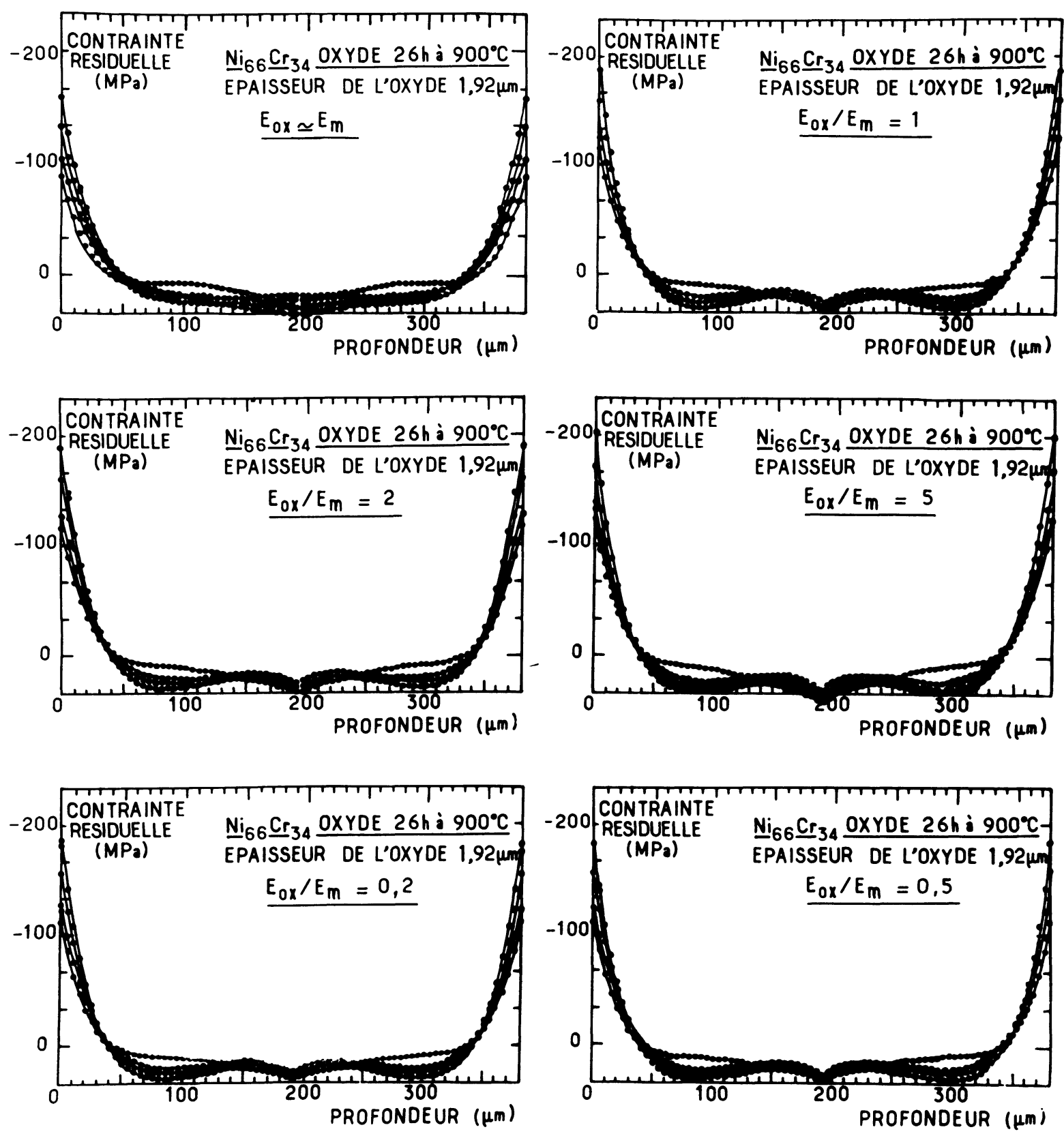

Fig. 12. - Distribution des contraintes dans l'épaisseur d'un échantillon $\mathrm{Ni}_{66} \mathrm{Cr}_{34}$ oxydé $26 \mathrm{~h}$ à $900{ }^{\circ} \mathrm{C}$. La présentation est identique à celle de la figure 7.

[Stress distribution in a $\mathrm{Ni}_{66} \mathrm{Cr}_{34}$ sample oxidized for $26 \mathrm{~h}$ at $900{ }^{\circ} \mathrm{C}$. Presentation similar to figure 7.]

Fig. 13. - Distribution des contraintes internes au voisinage de la surface externe et de l'interface M-oxyde. La notation du rapport des modules d'Young est analogue à celle utilisée dans la figure 7 .

a) $\mathrm{Ni}_{66} \mathrm{Cr}_{34}$ oxydé $48 \mathrm{~h}$ à $900^{\circ} \mathrm{C}$ sous $P_{\mathrm{O}_{2}}=3,5 \mathrm{~atm}$;

b) oxydation de $48 \mathrm{~h}$ à $900{ }^{\circ} \mathrm{C}$ sous $P_{\mathrm{O}_{2}}$ variable et décroissante ;

c) oxydation à $900{ }^{\circ} \mathrm{C}$ de $48 \mathrm{~h}$ sous $P_{\mathrm{O}_{2}}=1,4 \mathrm{~atm}$ et de $26 \mathrm{~h}$ sous $P_{\mathrm{O}_{2}}=2,5 \mathrm{~atm}$, de façon à obtenir une épaisseur d'oxyde voisine.

[Stress distribution near the oxidized sample outer surface. Notation similar to figure 7.

a) $\mathrm{Ni}_{66} \mathrm{Cr}_{34}$ oxidized for $48 \mathrm{~h}$ at $900^{\circ} \mathrm{C}$ with $P_{\mathrm{O}_{2}}=3.5 \mathrm{~atm}$;

b) $\mathrm{Ni}_{66} \mathrm{Cr}_{34}$ oxidized for $48 \mathrm{~h}$ at $900{ }^{\circ} \mathrm{C}$ with various decreasing $P_{\mathrm{O}_{2}}$;

c) $\mathrm{Ni}_{66} \mathrm{Cr}_{34}$ oxidized at $900{ }^{\circ} \mathrm{C}$ for $48 \mathrm{~h}$ with $P_{\mathrm{O}_{2}}=1.4 \mathrm{~atm}$, or $26 \mathrm{~h}$ with $P_{\mathrm{O}_{2}}=2.5 \mathrm{~atm}$, so that the oxide scale thickness has fairly the same value.] 

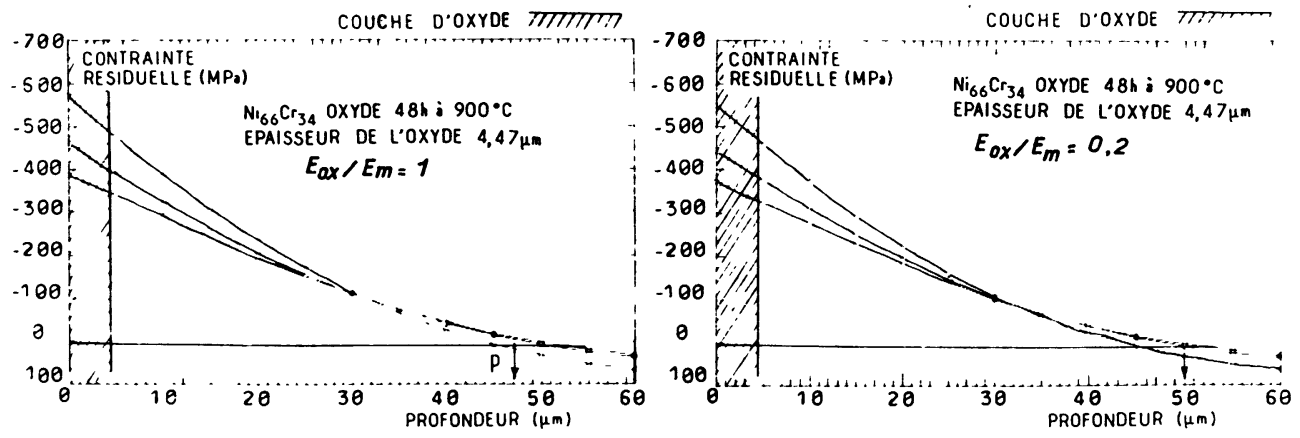

COUCHE DOXYOE TITITZ

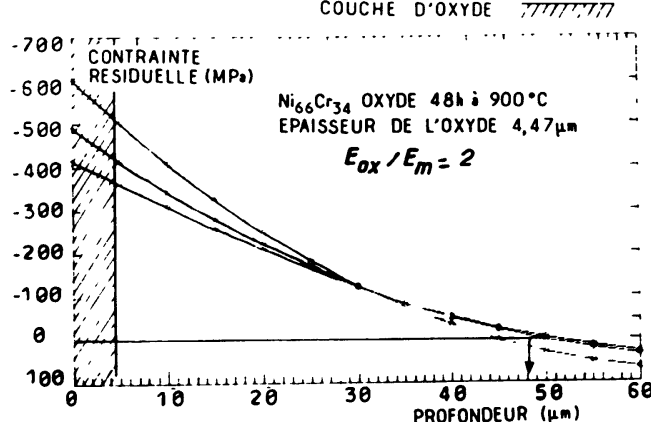

COUCHE D.OXYDE TIITITT

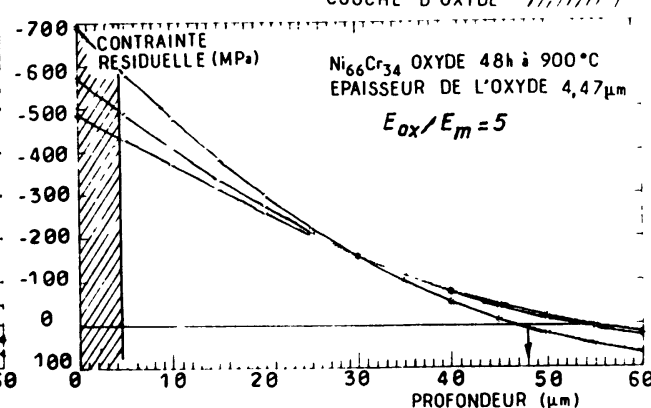

a)
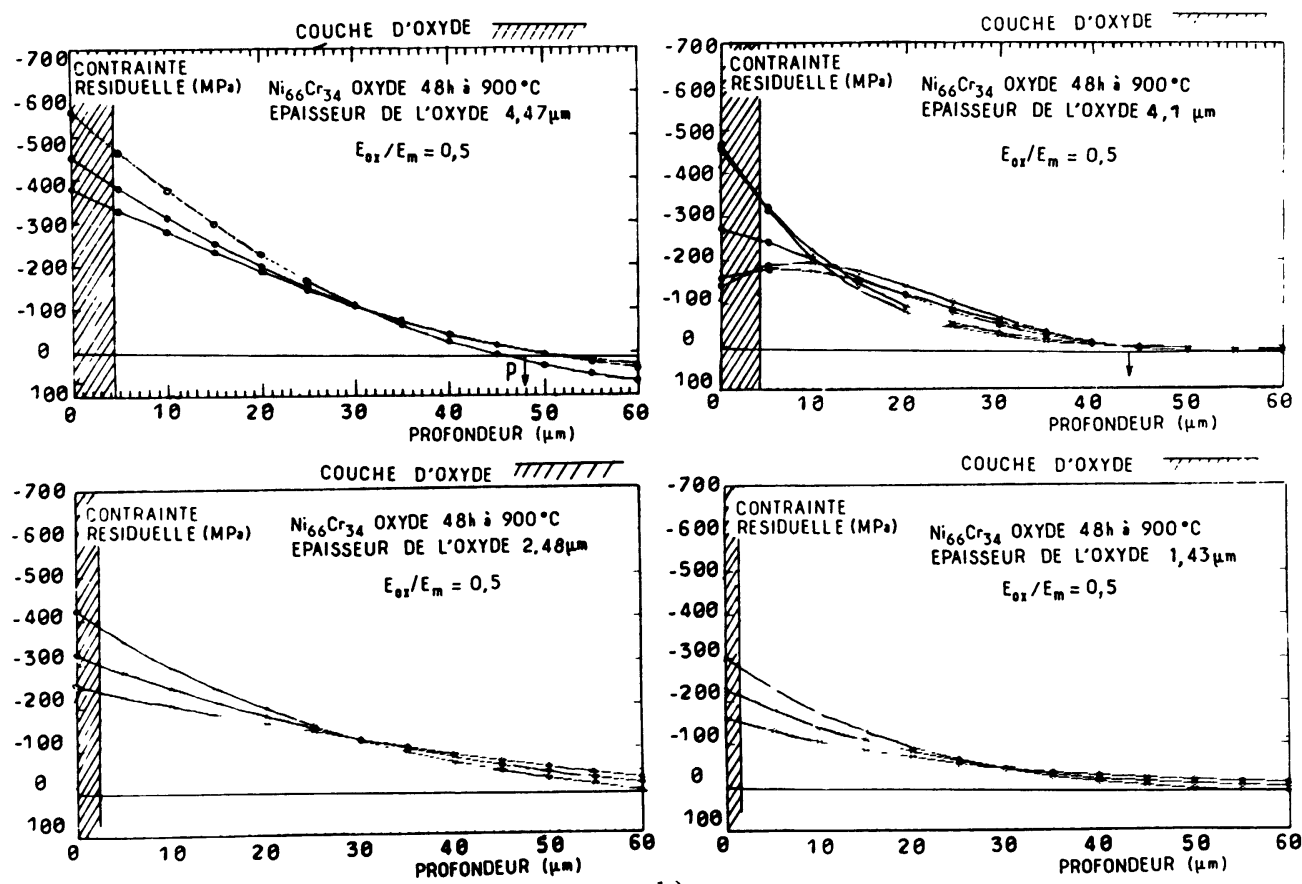

b)
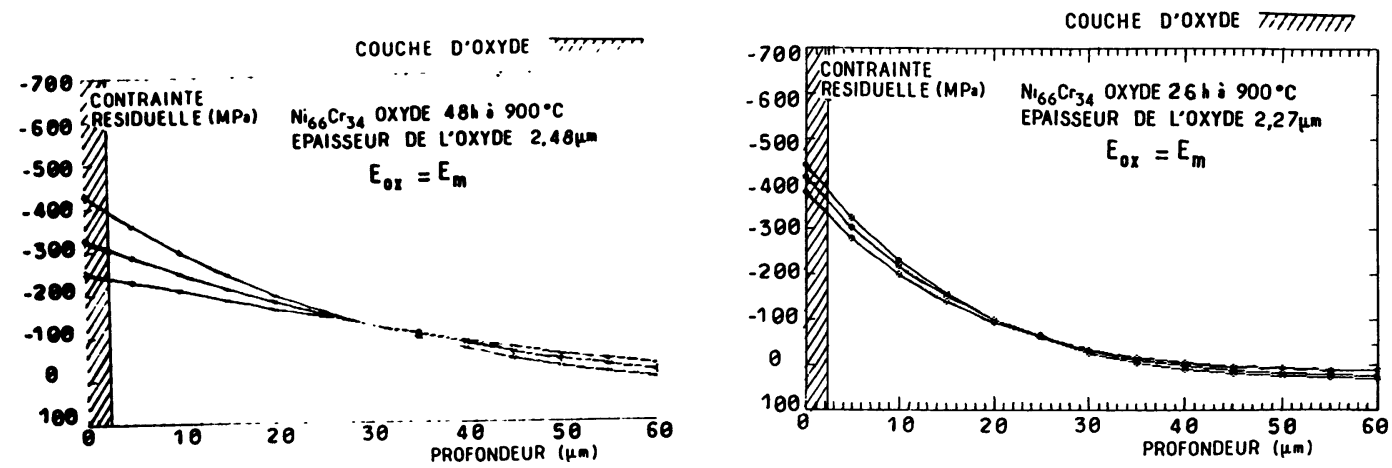

c) 
distribution des contraintes sont portées sur les figures 7 et 12 ; sur ces figures, les conditions d'oxydation et l'épaisseur de la couche d'oxyde sont indiquées en haut à droite. Pour la première figure (en haut à gauche), où il est indiqué $E_{\mathrm{ox}} \sim E_{\mathrm{m}}$, la distribution des contraintes a été calculée par la formule (9). Pour les autres figures $\sigma(h)$ a été calculée par la formule (13) et la valeur choisie pour le rapport $r=E_{\mathrm{ox}} / E_{\mathrm{m}}$ y est indiquée. Chaque figure contient au moins trois courbes de distribution des contraintes ; elles correspondent aux résultats obtenus avec les diverses courbes de lissage (cf. Fig. 6) ; c'est la valeur moyenne qui a un sens significatif. Nous constatons qu'il y a peu de différences entre les deux formulations utilisées.

Par ailleurs, le substrat métallique des échantillons est en compression pour les couches superficielles et légèrement en traction dans le cœur du substrat. La contrainte interne varie très vite près de la surface. Il n'existe pas de discontinuité près de l'interface $\mathrm{M}-\mathrm{O}$. Les couches d'oxyde sont soumises à des contraintes de compression de niveau assez élevé.

Ces mêmes résultats sont représentés dans les figures 13 après une dilatation de l'abscisse : l'épaisseur correspondant à la couche d'oxyde est indiquée par les zones hachurées.

Ces courbes nous permettent de déterminer la contrainte moyenne dans l'oxyde $\bar{\sigma}_{\text {ox }}$ et la profondeur $p$ de substrat soumis à des contraintes de compression. Nous pouvons ainsi comparer l'évolution de la distribution des contraintes internes d'oxydation en fonction de l'épaisseur de la couche d'oxyde $\left(\xi_{\text {ox }}\right)$. La relation entre $\bar{\sigma}_{\text {ox }}$ et $\xi_{\text {ox }}$ est présentée dans la figure 14 pour quatre valeurs du rapport $r\left(=E_{\mathrm{ox}} / E_{\mathrm{m}}\right)$ et celle entre $p$ et $\xi_{\mathrm{ox}}$ dans la figure 15 .

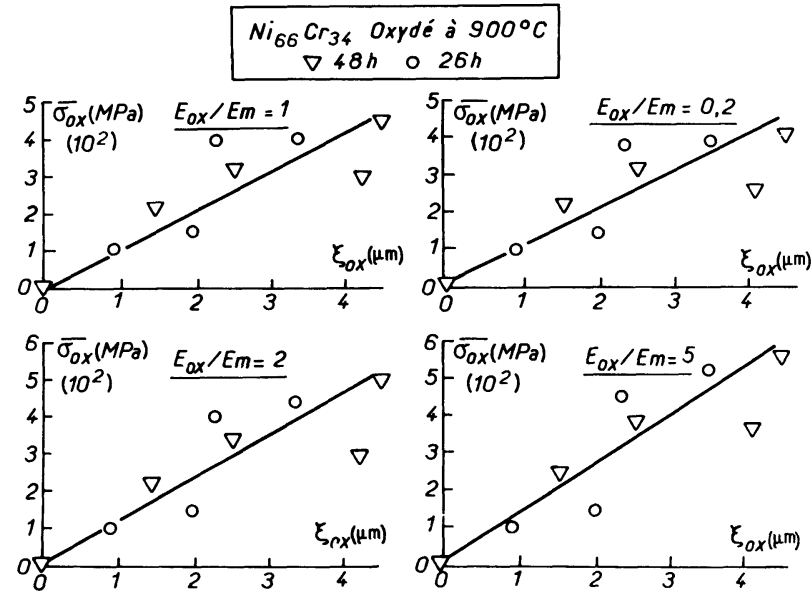

Fig. 14. - Variation de la contrainte interne moyenne dans la couche d'oxyde en fonction de son épaisseur pour quatre valeurs de $r\left(E_{\mathrm{ox}} / E_{\mathrm{m}}\right)$.

[Variation of the oxide scale average stress $v s$. the scale thickness, for various values of $r\left(E_{\mathrm{ox}} / E_{\mathrm{m}}\right)$.]

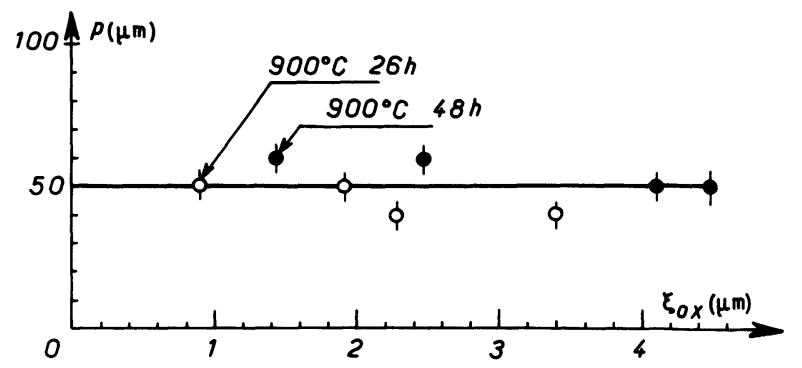

Fig. 15. - Variation de la profondeur $p$ de substrat soumis à des contraintes de compression, en fonction de l'épaisseur de la couche d'oxyde.

[Variation of the substrate depth $(p)$ submitted to compressive stresses $v s$. the oxide scale thickness.]

Nous constatons qu'il existe une relation linéaire entre le niveau de contraintes résiduelles dans l'oxyde et l'épaisseur de la couche d'oxyde (Fig. 14) : $\bar{\sigma}_{\text {ox }}$ augmente avec $\xi_{\text {ox }}$. Par ailleurs, le niveau de contraintes $\bar{\sigma}_{\text {ox }}$ augmente, mais faiblement, avec le rapport des modules d'Young $\left(r=E_{\text {ox }} / E_{\mathrm{m}}\right): \bar{\sigma}_{\text {ox }}$ augmente seulement de $10 \%$ lorsque $r$ passe de 0,2 à 5 . Quant à la valeur de $p$, elle varie peu avec les valeurs de $r$ (Fig. 15); l'épaisseur $\xi_{\text {ox }}$ n'a pas non plus d'influence notable sur la valeur de $p$ qui se situe aux alentours de $50 \mu \mathrm{m}$.

Il apparaît aussi que pour un temps d'oxydation donné, par exemple $48 \mathrm{~h}$ à $900^{\circ} \mathrm{C}$, sous des $P_{\mathrm{O}_{2}}$ variables, les contraintes de compression dans l'oxyde et dans le substrat sont d'autant plus importantes que la couche d'oxyde est épaisse (Fig. 13b). Enfin, pour une même épaisseur de couche d'oxyde, plus le traitement d'oxydation est rapide, plus les contraintes de compression dans le substrat sont élevées (Fig. 13c). Ce résultat est en accord avec ceux obtenus par analyse des contraintes par diffraction des RX [7], et indique que les possibilités de relaxation du substrat jouent un rôle important sur les contraintes résiduelles.

L'ensemble de ces résultats expérimentaux montre donc que la couche d'oxyde est vraiment soumise à des contraintes de compression de niveau élevé, que le cœur du substrat est soumis à de faibles contraintes de traction tandis que, vers l'interface $\mathrm{M}-\mathrm{O}$, le substrat est soumis à des contraintes de compression. Ils confirment donc les résultats obtenus antérieurement par diffraction $X$ et montrent tout l'intérêt de l'utilisation d'une telle technique. Néanmoins, il serait nécessaire de préciser ces phénomènes en opérant avec des abrasions beaucoup plus faibles; en effet, dans le travail actuel, la première couche enlevée par abrasion mécanique est importante et ne permet pas de déceler úne discontinuité dans la distribution des contraintes internes d'oxydation à l'interface $\mathrm{M}-\mathrm{O}$, discontinuité due à la différence des modules de Young du film et du substrat [8]. 


\section{Conclusions.}

Il est possible de déterminer la distribution des contraintes dans un système duplex constitué d'un substrat métallique et de sa couche d'oxyde par la méthode de la courbure.

Cette méthode consiste à mesurer l'évolution de la courbure d'un échantillon mince préalablement oxydé au cours d'abrasions successives de l'une de ses grandes faces. La distribution des contraintes est établie en mesurant avec précision l'épaisseur résiduelle et la courbure de l'échantillon au cours des abrasions successives. La technique est relativement facile à mettre en œuvre mais l'obtention de résultats reproductibles nécessite beaucoup de soin.

Appliquée à l'étude des contraintes dans le système $\mathrm{NiCr}-\mathrm{Cr}_{2} \mathrm{O}_{3}$, elle a permis de confirmer des résultats obtenus par diffraction des $\mathrm{RX}$ aux grands angles, dont certains pouvaient prêter à controverse. En particulier, nous avons confirmé que le film d'oxyde est bien soumis à des compressions, et nous avons montré que le substrat est également soumis à des compressions, du moins sur une certaine épaisseur au voisinage de l'interface.

Ainsi, malgré la grande incertitude de mesure $(\approx 20 \%)$ sur le niveau exact de contraintes internes, la méthode de la courbure s'avère satisfaisante dans le cadre de l'étude des relations entre les contraintes internes d'oxydation et le niveau d'oxydation. Il est à noter, néanmoins, que cette technique permet seulement de déterminer les contraintes résiduelles après refroidissement, c'est-à-dire la somme des contraintes dues à l'oxydation et de celles dues à la différence des coefficients d'expansion de l'oxyde et du substrat, différence qui provoque la création de contraintes pendant le refroidissement.

\section{Annexe.}

EXEMPLE DE CORRECTIONS LIÉES AUX CONTRAINTES DE POLISSAGE. - Dans le cas de l'alliage $\mathrm{Ni}_{66} \mathrm{Cr}_{34}$, recuit $48 \mathrm{~h}$ sous vide puis poli avec du papier au SiC (grade 1200), dans le sens longitudinal, $\bar{\sigma}_{\mathrm{p}} \cdot \delta=-734 \mathrm{MPa} . \mu \mathrm{m} ;$ avec $E_{\mathrm{m}}=207500 \mathrm{~Pa}$, nous avons donc :

$$
F_{\mathrm{p}}=\frac{1}{h^{2}} \times 0,021224\left(\mu \mathrm{m}^{-1}\right) \quad(h \text { en } \mu \mathrm{m}) .
$$

Dans les mesures expérimentales, nous avons obtenu :

$\begin{array}{lrllllll} & h(\mu \mathrm{m}) & 380 & 320 & 280 & 250 & 160 & 125 \\ F & \left(10^{-6} \mu \mathrm{m}^{-1}\right) & 0,4 & 0,993 & 1,829 & 1,76 & 2,071 & 2,742 \\ F_{\mathrm{p}} & \left(10^{-6} \mu \mathrm{m}^{-1}\right) & - & 0,207 & 0,271 & 0,340 & 0,829 & 1,358 \\ F & \left(10^{-6} \mu \mathrm{m}^{-1}\right) & 0,4 & 1,2 & 2,1 & 2,1 & 2,9 & 4,1 \\ & (\text { corrigée) } & & & & & & \end{array}$

\section{Bibliographie}

[1] ZhaO, J. G., Huntz, A. M., Couffin, P., BARon, J. L., Acta Metall. 34 (1986) 1351.

[2] Zhao, J. G., Huntz, A. M., Lebrun, J. L., Proceedings du $8^{\mathrm{e}}$ congrès Européen de Corrosion, Nice (1985), p. 15, Ed. Cefracor.

[3] Flavenot, J. F., Niku LaRi, A., Les Mémoires Techniques du CETIM (1977) 31.

[4] Timoshenko, S. P., Young, D. H., Elements of Strength of Materials, 5th Edition (1968).
[5] Maeder, G., Rev. Fr. Méc. 2 (1982) 57.

[6] Huntz, A. M., Zhao, J. G., Proceedings of the Int. Conf. on Residual Stresses, Garmisch-Partenkirchen, FRG (1986).

[7] Huntz, A. M., ZhaO, J. G., Proceedings of the Int. Symp. on High Temperature Corrosion, Marseille (1986). Mater. Sci. Eng. 88 (1987) 213-219.

[8] Taniguchi, S., Trans. Iron Steel Inst. Jpn 25 (1985) 3. 